\title{
ON THE MINIMIZING PROPERTY OF THE HARMONIC FUNCTION
}

\author{
BY E. J. MCSHANE
}

1. Introduction. Let $D$ be a bounded connected open set and $D^{*}$ its boundary and let $\bar{D}=D+D^{*}$. It is well known $\dagger$ that if the function $u(x, y)$ be harmonic on $D$, then $u(x, y)$ minimizes the Dirichlet integral

$$
I[f]=\iint_{D}\left[f_{x}^{2}+f_{y}^{2}\right] d x d y
$$

in the class of all functions $f(x, y)$ possessing piecewise continuous partial derivatives $f_{x}$ and $f_{y}$ and coinciding with $u(x, y)$ on the boundary $D^{*}$. But in certain recent discussions of the problem of Plateau $\ddagger$ essential use is made of a generalization of this theorem; it was necessary to know that the harmonic function $u(x, y)$ minimizes $I[f]$ in a larger class of functions than those with continuous derivatives. It has been suggested that a proof of this fact should be published; the present note carries out the suggestion. The method of proof is similar to that due to Lebesgue. As an application, a theorem is proved which is of some interest in the theory of curved surfaces.

The functions with which we shall be concerned are those which are, as we shall say, absolutely continuous by sections (abbreviated a.c.s.). A function $v(x, y)$, defined and continuous on a bounded open set $D$, will be said to be a.c.s. on $D$ if it satisfies the following conditions:

(1 a) for almost all values $y_{0}$ of $y$ the function $v\left(x, y_{0}\right)$ is absolutely continuous on each interval of the line $y=y_{0}$ lying in $D$;

(1 b) for almost all values $x_{0}$ of $x$ it is absolutely continuous on each interval of the line $x=x_{0}$ lying in $D$.

$\dagger$ H. Lebesgue, Société Mathématique de France, Comptes Rendus, (1913), p. 48. Hurwitz-Courant, Funktionentheorie, 2d ed., p. 424.

$\ddagger$ E. J. McShane, Parametrizations of saddle surfaces, etc., Transactions of this Society, vol. 35 (1933), pp. 716-733. T. Rad6, The problem of Plateau, vol. II, No. 3, of the Ergebnisse der Mathematik und ihre Grenzgebiete, p. 99. 
With this terminology we state our theorem on the minimizing property of the harmonic function.

Theorem 1. If (a) $u$ is continuous on $\bar{D}$ and harmonic on $D$, (b) $v$ is continuous on $\bar{D}$ and a.c.s. on $D$, (c) $v(x, y)=u(x, y)$ on $D^{*}$, then $I[v] \geqq I[u]$, the sign of equality holding only if $I[u]=\infty$ or $v \equiv u$.

Before proceeding to the proof of the theorem, we first observe that if $f(x, y)$ be a.c.s. on $D$, its derivatives $v_{x}$ and $v_{y}$ are defined almost everywhere on $D$ and are measurable where defined. Where they are undefined, we assign them the value 0 . We shall denote by $X$ the set of all values $x_{0}$ of $x$ such that (a) the line $x=x_{0}$ has points in common with $D$, (b) on each interval of the line $x=x_{0}$ lying in $D$ the function $v\left(x_{0}, y\right)$ is absolutely continuous in $y$. The set $Y$ is defined analogously. For each $x_{0}$ of $X$ the line $x=x_{0}$ has in common with $D$ a finite or denumerable set of intervals; these we denote by $\delta_{1}\left(x_{0}\right), \delta_{2}\left(x_{0}\right), \cdots$. The intervals $\delta_{i}\left(y_{0}\right)$ are analogously defined. As a first step in the proof of Theorem 1 we establish the following lemma.

Lemma. If $u(x, y)$ is harmonic on an open set containing $\bar{D}$, and $v$ is continuous on $\bar{D}$ and a.c.s. on $D$, and $v(x, y)=u(x, y)$ on $D^{*}$, then $I[v] \geqq I[u]$.

If $I[v]=\infty$, the conclusion holds. Otherwise let us define

$$
\phi(x, y)=v(x, y)-u(x, y) .
$$

This function is continuous on $\bar{D}$ and a.c.s. on $D$, and

$$
I[v]=I[u]+I[\phi]+2 \iint_{D}\left(u_{x} \phi_{x}+u_{y} \phi_{y}\right) d x d y ;
$$

here all integrals are well defined and finite. We may write

$$
\begin{aligned}
\iint_{D} u_{x} \phi_{x} d x d y & =\int_{Y}\left\{\int_{\Sigma \delta_{\boldsymbol{t}}(y)} u_{x} \phi_{x} d x\right\} d y \\
& =\int_{Y}\left\{\sum \int_{\delta_{i}(y)} u_{x} \phi_{x} d x\right\} d y .
\end{aligned}
$$

In the last integral we integrate by parts, remembering that $\phi$ vanishes at each end of each $\delta_{i}(y)$. We thus obtain 


$$
\begin{aligned}
\iint_{D} u_{x} \phi_{x} d x d y & =-\int_{Y}\left\{\sum \int_{\delta_{i}(y)} u_{x x} \phi d x\right\} d y \\
& =-\iint_{D} u_{x x} \phi d x d y ;
\end{aligned}
$$

the last reduction is allowable because $u_{x x}$ and $\phi$ are both continuous and bounded on $\bar{D}$. In a like manner,

$$
\iint_{D} u_{y} \phi_{y} d x d y=-\iint_{D} u_{y y} \phi d x d y .
$$

Adding and remembering that $u$ is harmonic, we find that the last integral of equation (1) vanishes. Since $I[\phi] \geqq 0$, the lemma is established.

Proceeding to the proof of the theorem, we subdivide $D$ into the sets $D_{0}, D^{\prime}, D^{\prime \prime}$, on which the respective relations $v=u$, $v>u, v<u$ hold. Also, for every $\epsilon>0$, we define $D_{\epsilon}^{\prime}$ and $D_{\epsilon}^{\prime \prime}$ to be the subsets of $D$ on which $v>u+\epsilon$ and $v<u-\epsilon$, respectively. The Dirichlet integrals over these sets will be distinguished by the corresponding affixes; for example, $I_{\epsilon}^{\prime}(u)$ is the integral of $u_{x}^{2}+u_{y}^{2}$ over $D_{\epsilon}^{\prime}$.

We first observe that $I_{0}[v]=I_{0}[u]$; in fact, $u_{x}=v_{x}$ and $u_{y}=v_{y}$ for almost all points of $D_{0}$. For let $E$ be the set on which $u-v=0$, the derivatives $u_{x}$ and $v_{x}$ are defined, and $u_{x}-v_{x} \neq 0$. This set is measurable; hence to prove that its measure is zero, we need only show that for almost all $y_{0}$ the part of $E$ lying on the line $y=y_{0}$ has linear measure zero. But for every $y_{0}$ the points of $E$ belonging to the line $y=y_{0}$ form an isolated set and so are enumerable; for at each such point we have $u-v=0$, while $\partial(u-v) / \partial x$ exists and is not zero. Therefore $m(E)=0$.

As $\epsilon$ tends to 0 , the sets $D_{\epsilon}^{\prime}, D_{\epsilon}^{\prime \prime}$ tend to $D^{\prime}, D^{\prime \prime}$, respectively; hence $I_{\epsilon}^{\prime}[u] \rightarrow I^{\prime}[u]$ and $I_{\epsilon}^{\prime \prime}[u] \rightarrow I^{\prime \prime}[u]$. Thus if $h$ be any number less than $I[u]$, we can choose $\epsilon$ small enough so that $I_{\epsilon}^{\prime}[u]+I_{\epsilon}^{\prime \prime}[u]+I_{0}[u]>h$. The set $D_{\epsilon}^{\prime}$ lies, with its boundary $D_{\epsilon}^{\prime *}$, in $D$; and on $D_{\epsilon}^{\prime *}$ we have $v-\epsilon=u$. Hence by our lemma $I_{\epsilon}^{\prime}[v]=I_{\epsilon}^{\prime}[v-\epsilon] \geqq I_{\epsilon}^{\prime}[u]$, and similarly $I_{\epsilon}^{\prime \prime}[v] \geqq I_{\epsilon}^{\prime \prime}[u]$. Thus

$$
I[v] \geqq I_{0}[v]+I_{\epsilon}^{\prime}[v]+I_{\epsilon}^{\prime \prime}[v] \geqq I_{0}[u]+I_{\epsilon}^{\prime}[u]+I_{\epsilon}^{\prime \prime}[u]>h \text {. }
$$

This being true for every $h<I[u]$, it follows that $I[v] \geqq I[u]$, as was to be proved. 
It remains to be shown that if $I[u]$ is finite and $I[v]=I[u]$, then $v \equiv u$. In this case we consider the function

$$
I[u+\lambda \phi]=I[u]+\lambda^{2} I[\phi]+2 \lambda \iint_{D}\left(u_{x} \phi_{x}+u_{y} \phi_{y}\right) d x d y,
$$

where $\phi=v-u$. On setting $\lambda=1$, we find that the last integral on the right has the value $-I[\phi] / 2$. Setting $\lambda=1 / 2$, the equation becomes $I[u+\phi / 2]=I[u]-I[\phi] / 4$. Therefore, if $I[\phi]>0$, the a.c.s. function $u+\phi / 2$ has a Dirichlet integral less than that of $u$, which we have already shown impossible; hence $I[\phi]=0$. This implies that $\phi_{x}=0$ almost everywhere in $D$. Thus for almost all $y_{0}$ of $Y$ the equation $\phi_{x}\left(x, y_{0}\right)=0$ holds for almost all $x$ in $\sum \delta_{i}\left(y_{0}\right)$; integrating and remembering that $\phi=0$ at the ends of each $\delta_{i}\left(y_{0}\right)$, we find $\phi(x, y)=0$ for almost all $(x, y)$ in $D$. Since $\phi$ is continuous, $\phi=u-v$ vanishes identically on $D$, completing the proof of the theorem.

2. Discussion of a.c.s. Functions. As yet we have not shown that the class of a.c.s. functions includes the class of functions with piecewise continuous derivatives and finite Dirichlet integral. Suppose, then, that $v(x, y)$ and its derivatives $v_{x}$ and $v_{y}$ are continuous on $D$, and $I[v]$ is finite; we state that $v$ is a.c.s. For the finiteness of $I[v]$ implies that $\left|v_{x}\left(x, y_{0}\right)\right|$ is summable over $\sum \delta_{i}\left(y_{0}\right)$ for almost all $y_{0}$. Since $v_{x}$ is continuous in $D, v$ is absolutely continuous over every closed interval contained in $\delta_{i}\left(y_{0}\right)$; and this, with the summability of $v_{x}\left(x, y_{0}\right)$, implies that $v$ is absolutely continuous on $\delta_{i}\left(y_{0}\right)$. A like argument applies to $v\left(x_{0}, y\right)$ for almost all $x_{0}$. In particular, if $u(x, y)$ is harmonic on $D$ and $I[u]$ is finite, then $u(x, y)$ is a.c.s.

If $v$ is continuous on $D$ and $I[v]$ is finite, and $D$ can be subdivided into a finite number of subsets, each bounded by a finite number of rectifiable simple arcs such that $v_{x}$ and $v_{y}$ are continuous on the interior of each subset, then $v$ is a.c.s. For, if we except a set of $x_{0}$ of measure zero, the line $x=x_{0}$ intersects the boundary curves in a finite number of points, so that each $\delta_{i}\left(x_{0}\right)$ is subdivided into a finite number of sub-intervals. Again except on a set of measure zero, the function $v\left(x_{0}, y\right)$ is absolutely continuous on each of these subintervals by the preceding paragraph, and being also continuous on $\delta_{i}\left(x_{0}\right)$ it must be absolutely continuous on $\delta_{i}\left(x_{0}\right)$. A like argument holds for almost all $y_{0}$. 
3. Invariance Properties. The definition of the property a.c.s. seems highly artificial, since it is stated in terms of a particular coordinate system and has no self-evident invariance properties even under rotations of the axes. But this defect is only apparent; in fact, we shall now apply Theorem 1 to show that if a function is a.c.s. and has a finite Dirichlet integral, it retains these properties under all conformal transformations, the Dirichlet integral being unchanged in value. We shall prove the following theorem.

\section{THEOREM 2. Let the transformation}

$$
x=x\left(x^{\prime}, y^{\prime}\right), y=y\left(x^{\prime}, y^{\prime}\right)
$$

map the bounded open set $D^{\prime}$ conformally on the bounded open set $D$ and map $\bar{D}^{\prime}$ topologically on $\bar{D}$, and let the function $v^{\prime}\left(x^{\prime}, y^{\prime}\right)$ be a.c.s. on $D^{\prime}$ and have a finite Dirichlet integral

$$
I^{\prime}\left[v^{\prime}\right] \equiv \iint_{D^{\prime}}\left[{v^{\prime}}^{2}{ }_{x^{\prime}}+{v^{\prime}}_{y^{\prime}}^{2}\right] d x^{\prime} d y^{\prime}
$$

Then its transform $v(x, y)=v^{\prime}\left(x^{\prime}(x, y), y^{\prime}(x, y)\right)$ is continuous on $\bar{D}$ and a.c.s. on $D$, and $I[v]=I^{\prime}\left[v^{\prime}\right]$.

By drawing lines parallel to the $x$ - and $y$-axes we subdivide the $(x, y)$-plane into squares of side $2^{-n}$, where $n$ is an arbitrary positive integer. We obtain the $(n+1)$ th subdivision by subdividing each square of the $n$ th. Those squares which are (with their boundaries) interior to $D$ we call $q_{1}, q_{2}, \cdots, q_{m}$; the remainder of $D$ is a set $r$, bounded in part by $D^{*}$ and in part by line segments. In the $q_{j}$ and in $r$ we construct the harmonic functions which coincide with $v(x, y)$ on the boundaries of the subsets. Thus we have defined a function $u_{n}(x, y)$ which is continuous on $D$ and harmonic on the interiors of the $q_{j}$ and $r$. The transformation (3) carries $r, q_{1}, q_{2}, \cdots, q_{m}$ into subsets of $D^{\prime}$, which we call $r^{\prime}, q_{1}{ }^{\prime}, q_{2}{ }^{\prime}, \cdots, q_{m}{ }^{\prime}$, respectively; and it carries $u_{n}^{\prime}$ into a function $u_{n}^{\prime}\left(x^{\prime}, y^{\prime}\right)$ which is continuous on $D^{\prime}$ and harmonic on the interior of each $q_{j}^{\prime}$ and $r^{\prime}$ and which coincides with $v^{\prime}$ on the boundary of each subset. Hence, by Theorem 1 , the Dirichlet integral of $v_{n}^{\prime}$ over each subset is at most equal to the Dirichlet integral of $u^{\prime}$ over that set, so on adding these integrals we have $I^{\prime}\left[u_{n}^{\prime}\right] \leqq I^{\prime}\left[v^{\prime}\right]$. But in each set $q_{i}, r$, the map- 
ping (3) leaves the Dirichlet integral of the harmonic function $u$ unchanged; hence $I\left[u_{n}\right]=I^{\prime}\left[u_{n}^{\prime}\right] \leqq I^{\prime}\left[v^{\prime}\right]$. Let us consider a point $\left(x_{0}, y_{0}\right)$ of $D$. For all sufficiently large $n$, it belongs to some square, say $q_{i(n)}$, of the $n$th subdivision. Since the diameters of the squares approach zero, the greatest and least of the values of $v(x, y)$ on the boundary of $q_{i(n)}$ tend to $v\left(x_{0}, y_{0}\right)$; and since $u_{n}$ is harmonic on the square, the value $u_{n}\left(x_{0}, y_{0}\right)$ lies between these two extreme values of $v$, so that $\lim u_{n}(x, y)=v(x, y)$ at each point of $D$. This equation continues to hold on $D^{*}$, where every $u_{n}$ coincides with $v$. Finally, by $\S 2$, each $u_{n}(x, y)$ is a.c.s. on $D$.

We are now in a position to repeat the demonstration on pages 719-720 of my previously cited paper; we need only replace the word "uniformly" by "everywhere" and replace the interated integrals taken first with respect to $v$ between $-\left(1-u^{2}\right)^{1 / 2}$ and $\left(1-u^{2}\right)^{1 / 2}$ and then with respect to $v$ over $C E$, by iterated integrals taken first over $\sum \delta_{i}(x)$ and then over $X$. We thus find that $v(x, y)$ is a.c.s., and that

$$
I[v] \leqq \lim \inf I\left[u_{n}\right]=\lim \inf I^{\prime}\left[u_{n}^{\prime}\right] \leqq I^{\prime}\left[v^{\prime}\right] .
$$

Interchanging the roles of $v$ and $v^{\prime}$ shows that $I^{\prime}\left[v^{\prime}\right] \leqq I[v]$; hence the Dirichlet integrals are equal, and the theorem is established. This theorem has an application in the theory of curved surfaces. Among all continuous surfaces

$$
x=x(u, v), \quad y=y(u, v), \quad z=z(u, v), \quad((u, v) \text { on } B),
$$

where $B$ is a Jordan region, a particularly interesting class is that for which

$(\alpha)$ the functions $x(u, v), y(u, v), z(u, v)$ are a.c.s. on $B$,

( $\beta) E+G$ is summable, that is, $I[x], I[y], I[z]$ are finite.

In particular, if the region $B$ can be taken to be a circle, $\alpha$ and $\beta$ remaining satisfied, we say (with Morrey) that the surface is of type $L_{2}$. It is clearly more convenient to have a circle to deal with than to have a general Jordan region. But we can now show that every surface having a representation satisfying $(\alpha),(\beta)$ is of type $L_{2}$. We need only map $B$ conformally on the unit circle; the transformed functions $\bar{x}(u, v), \bar{y}(u, v), \bar{z}(u, v), u^{2}+v^{2} \leqq 1$, then satisfy $(\alpha)$ and $(\beta)$ because of Theorem 2 . 